\title{
Indicadores de desenvolvimento humano e de saúde bucal na atenção básica nos municípios da região metropolitana de Curitiba-PR
}

\author{
Human Development Indicators and oral health indicators in Primary \\ Care in the municipalities of Metropolitan Region of Curitiba-PR
}

\author{
Thábata Cristy Zermiani \\ Bárbara Vieira Pimentel* \\ Marilene da Cruz Magalhães Buffon** \\ Rafael Gomes Ditterich ${ }^{* * *}$
}

\section{Resumo}

Objetivo: o presente estudo tem como objetivo monitorar as ações em saúde bucal dos municípios do Núcleo Urbano Central (NUC) da Região Metropolitana de Curitiba - Paraná, por meio do uso de sistemas de informação, verificando a existência de correlação entre indicadores da atenção primária em saúde bucal e os de desenvolvimento humano. Materiais e método: o estudo classifica-se como descritivo correlacional, com avaliação de dados secundários. Os indicadores de desenvolvimento humano utilizados foram o Índice de Desenvolvimento Humano $\mathrm{Mu}$ nicipal (IDHM) e o Índice Ipardes de Desempenho Municipal (IPDM), obtidos na base de dados disponibilizada pelo Programa das Nações Unidas para o Desenvolvimento (PNUD) e pelo Instituto Paranaense de Desenvolvimento Econômico e Social (Ipardes), ambos referentes ao ano de 2010. Os indicadores em saúde bucal foram acessados e analisados pela produção ambulatorial do Sistema de Informação em Saúde do Sistema Único de Saúde (SIA-SUS) e consistem, na consolidação do ano de 2012 em relação a: a) primeira consulta odontológica programática; b) ação coletiva de escovação dental supervisionada; c) procedimentos odontológicos individuais básicos; e d) exodontias de decíduos e permanentes. $\mathrm{Na}$ análise estatística quantitativa, utilizou-se o teste de correlação de Pearson, com nível de significância de 95\%. Resultados: a comparação dos dados explicitou que quanto maior o desenvolvimento humano, maior é o número de primeiras consultas odontológicas programáticas $(r=0,5645)$. Encontrou-se ainda a correlação entre o número de primeiras consultas e $o$ de exodontias $(r=0,6439)$. Conclusão: os sistemas de informação constituem-se em importantes ferramentas no planejamento local das ações de saúde bucal dos municípios.

Palavras-chave: Indicadores básicos de saúde. Sistemas de informação em saúde. Gestão da informação em saúde: saúde bucal. Sistema único de saúde.

Acadêmica do curso de graduação de Odontologia da Universidade Federal do Paraná (UFPR), Curitiba, Paraná, Brasil. Bolsista PET-Saúde / Redes de Atenção UFPR/SMS, Curitiba-PR, Brasil.

Doutora em Ciências pela Universidade Federal do Paraná (UFPR), professora Associada do Departamento de Saúde Comunitária da Universidade Federal do Paraná (UFPR), Curitiba-PR, Brasil.

Doutor em Odontologia (Saúde Coletiva) pela Pontifícia Universidade Católica do Paraná (PUCPR), professor adjunto do Departamento de Saúde Comunitária da Universidade Federal do Paraná (UFPR), Curitiba-PR, Brasil. 


\section{Introdução}

Com a implantação do Sistema Único de Saúde (SUS) pela constituição de 1988 a sua regulamentação pelas leis n. $8080 / 90$ e n. 8142/90, o conceito de saúde foi ampliado e o acesso a essa passou a ser considerado direito fundamental de todo cidadã $0^{1,3}$. Assim, o objetivo desse sistema consiste em alcançar a universalização do acesso, a integralidade das ações, a equidade, a descentralização, a hierarquização dos serviços e o controle social. A consolidação do SUS fundamentou-se na reorientação da atenção básica, por meio do Programa de Agentes Comunitários (PACS) e do Programa Saúde da Família (PSF), o qual em 2011 passou a ser designado Estratégia Saúde da Família (ESF) ${ }^{4}$. O ESF tem como objetivo a reorganização da prática da atenção à saúde em novas bases, substituindo o modelo tradicional, considerando permanentemente o meio e a forma de organização social na qual o indivíduo está inserido ${ }^{5}$.

A Equipe de Saúde Bucal foi, mais tarde, inserida no ESF, por meio da Portaria n. 267/2001, no entanto já se apresenta como parte integrante e importante para a saúde da população $0^{2,6}$. Sua inserção se deu com o propósito de mudança do modelo de atenção à saúde, visando melhorar as condições de saúde bucal da população e reorientar suas práticas, avaliando o padrão de qualidade e o impacto das ações desenvolvidas, ampliando o acesso coletivo às ações de promoção, de prevenção e de recuperação da saúde bucal.

A fim de orientar o desenvolvimento da programação e do planejamento das ações a serem realizadas, deve-se levar em consideração o modelo vigente de atenção em saúde bucal, o diagnóstico das condições de saúde e as necessidades de tratamento da população adscrita no território em questão. Dessa forma, pode-se estabelecer prioridades e direcionar os recursos necessários à modificação positiva das condições de saúde da população. A informação é, portanto, essencial à tomada de decisões e orienta quanto às ações a serem desenvolvidas, visando à promoção da saúde, à prevenção de agravos e à organização dos serviços oferecidos ${ }^{4}$.

Nesse contexto, evidencia-se a importância dos Sistemas de Informação em Saúde, como o Sistema de Informações Ambulatoriais do SUS (SIA-SUS), bem como dos bancos de dados sobre desenvolvimento social, como o Índice de Desenvolvimento Humano Municipal (IDH-M), o Índice Ipardes de Desenvolvimento Municipal (IPDM) e o censo para subsidiar a tomada de decisões relativas às ações a serem desenvolvidas ${ }^{4,6}$.

Os Sistemas de Informação constituem-se em unidades de produção, análise e disseminação de dados, objetivando subsidiar a elaboração e a avaliação das ações de saúde desenvolvidas, por meio da análise dos indicadores. Dentre os sistemas na- cionais, os únicos empregados pelas Equipes de Saúde Bucal, são: Sistema de Informação da Atenção Básica (SIAB) e SIA-SUS ${ }^{4,6,7}$.

O monitoramento contínuo desses indicadores é importante na medida em que instrui o direcionamento de recursos adicionais para as áreas com maiores necessidades, contribuindo para a implementação de serviços de saúde socialmente apropriados. A equidade deve ser uma prioridade no planejamento das ações de saúde e o desafio de mensurar essa dimensão deve continuar instigando os estudos em saúde bucal ${ }^{8}$.

Verifica-se que a análise dos indicadores coletados de Banco de Dados, principalmente do SIA-SUS, associados a dados socioeconômicos, como o IDH-M e IPDM é bastante significativa para a avaliação dos impactos das ações em saúde realizadas nos âmbitos municipal, estadual e federal, o que possibilita evitar o aumento da oferta de serviços sem planejamento adequado ${ }^{8,9}$.

O SIA-SUS foi implantado em 1991, substituindo o sistema de pagamento de serviços ambulatoriais até então vigente ${ }^{10}$. O Sistema contém os procedimentos realizados pelas unidades de saúde, inseridas ou não na Estratégia Saúde da Família. A produção é lançada mensalmente, incluindo as atividades em saúde bucal no âmbito da atenção básica ${ }^{6}$. $\mathrm{O}$ acompanhamento dessa produção e do impacto da utilização desses recursos, que são monitorados através do estabelecimento de metas pactuadas anualmente entre as três esferas do governo, constituem o Pacto de Atenção Básica. A consulta ao SIA-SUS permite, portanto, um acompanhamento da produção ambulatorial odontológica, assim como a busca de dados para efetuar o cálculo dos indicadores quantitativos odontológicos que auxiliam na avaliação da organização da saúde bucal nos municípios?

Para a realização da presente pesquisa foram utilizados os quatro indicadores, segundo a redefinição realizada pelo Pacto de Indicadores da Atenção Básica pela Portaria n. 493/GM de 10 de março de 2006:

a) Primeira consulta odontológica programática: relacionada ao percentual da população que recebeu uma primeira consulta odontológica programática com finalidade de diagnóstico elaborando um Plano Preventivo Terapêutico (PPT), no âmbito de um programa de saúde. Destaca-se que seu registro deve ser anual, ou seja, a primeira consulta realizada no ano pelo usuário do SUS e não a primeira consulta que determinado profissional realizou $4,7,11,12$.

b) Ação coletiva de escovação dental supervisionada: corresponde ao percentual da população que participou da ação coletiva escovação dental supervisionada. Essa ação é dirigida a um grupo de indivíduos, tendo por objetivo a prevenção de doenças bucais, de forma mais específica, a cárie e a doença periodontal $\left.\right|^{4,11,12}$.

c) Procedimentos odontológicos individuais básicos: consiste no número médio de procedimentos odontológicos básicos individuais, podendo ser cirúrgicos/clínicos, realizados por 
indivíduo na população residente em determinado local e período. Esse registro possibilita a estimativa de em que medida os serviços do SUS estão atendendo às necessidades de assistência odontológica básica da população ${ }^{11,12}$.

d) Exodontias de decíduos e permanentes: tem como objetivo incentivar as ações que não sejam mutiladoras ${ }^{12}$.

O Índice de Desenvolvimento Humano, criado por Mahbub ul Haq com a colaboração de Amartya Sem, tem o objetivo de oferecer um contraponto a outro indicador muito utilizado, o Produto Interno Bruto (PIB) per capita, que considera apenas a dimensão econômica do desenvolvimento. Em síntese, o IDH pretende ser uma medida geral do desenvolvimento humano ${ }^{13}$.

Atualmente, os três pilares que constituem o IDH (saúde, educação e renda) são mensurados através da expectativa de vida (saúde); do grau de escolaridade (educação); e do PIB per capita expresso em poder de paridade de compra (renda) ${ }^{8,13}$.

No Brasil, tem-se empregado também o Índice de Desenvolvimento Humano Municipal (IDH-M), o qual consiste em um ajuste metodológico do IDH Global, e foi publicado em 1998 - a partir dos dados dos Censos de 1970, 1980 e 1991 -, em 2003 - a partir dos dados do Censo de 2000 -, e 2013 - a partir dos dados do Censo de $2010^{13}$. Considerando que é um ajuste metodológico, não é possível fazer comparação entre o IDH-M de um município e o IDH de um país ${ }^{14}$.

O IDH-M é um número que varia entre 0 e 1 , quanto mais próximo de 1 , maior o desenvolvimento humano de um município. Os municípios com IDH-M até 0,499 apresentam desenvolvimento humano considerado muito baixo; IDH-M entre 0,500 e 0,599 é classificado como baixo; entre 0,600 e 0,699 é apontado como médio; entre 0,700 e 0,799 é tido como alto; e acima de 0,800 é considerado muito alto ${ }^{15}$.

No estado do Paraná, o Instituto Paranaense de Desenvolvimento Econômico e Social (Ipardes) desenvolveu o Índice Ipardes de Desenvolvimento Municipal (IPDM), o qual mede o desempenho da gestão e de ações públicas dos municípios paranaenses. Para tanto, considera três dimensões: renda, emprego e agropecuária, saúde e educaçãa ${ }^{16}$.

Tanto o índice geral como os parciais apresentam variações de 0 a 1 , números que representam, respectivamente, condições mínima e máxima de desempenho socioeconômico. Para auxiliar a leitura dos indicadores finais, esses são divididos entre os estágios de baixo $(0,000$ a 0,399$)$, médio-baixo $(0,400$ a 0,599$)$, médio $(0,600$ a 0799$)$ e alto desempenho $(0,800 \text { a } 1,000)^{17}$.

Considerando os valores do IDH-M e do IPDM torna-se possível avaliar o quadro de desenvolvimento de determinado município.

Observa-se uma forte correlação entre o IDH e os principais indicadores de saúde bucal ${ }^{18,19}$.

Moysés $^{20}$ (2000), em sua pesquisa, demonstrou que morar em um local com uma política pública saudável, a qual seja implementada de forma eficaz e que apresente um maior grau de coesão social tem um fator de proteção para a sua saúde bucal. Por exemplo, a proporção de dentes cariados e extraídos é nitidamente maior entre os municípios de baixo IDH, ocorrendo o inverso com a proporção de dentes restaurados. Dessa forma, a população de municípios de baixo IDH tem maior chance de ter dente cariado ou extraído, sendo que a proporção de perdas de elementos dentários em municípios de pior qualidade de vida é três vezes maior que em municípios com alto IDH ${ }^{18}$.

A redução dessas iniquidades em saúde bucal somente será alcançada com a implementação de políticas de promoção de saúde efetivas e apropriadas $^{21}$. Dessa forma, o objetivo deste trabalho foi monitorar as ações em saúde bucal dos municípios do Núcleo Urbano Central da Região Metropolitana de Curitiba-PR, por meio do uso dos Sistemas de Informações, verificando a existência de correlação entre os índices IDH-M e IPDM e os indicadores supracitados.

\section{Materiais e método}

A presente pesquisa consiste em um estudo descritivo correlacional, com a utilização de dados secundários quantitativos. A região delimitada para análise foi o Núcleo Urbano Central (NUC) de Curitiba-PR. Segundo a Coordenação de Região Metropolitana de Curitiba (Comec), essa área é composta por 14 municípios - Almirante Tamandaré, Araucária, Campina Grande do Sul, Campo Largo, Campo Magro, Colombo, Curitiba, Fazenda Rio Grande, Itaperuçu, Pinhais, Piraquara, Quatro Barras, Rio Branco do Sul e São José dos Pinhais ${ }^{22}$. Os municípios que compõem o NUC foram escolhidos por se tratarem de uma região consideravelmente urbanizada e, também, pelas diferenças de desenvolvimento socioeconômico entre esses.

Os indicadores de Saúde Bucal que foram utilizados nesta pesquisa foram: primeira consulta odontológica programática, escovação supervisionada, procedimentos individuais básicos e exodontias de decíduos e permanentes, os quais foram obtidos por meio de acesso público a base de dados do SIA-SUS, tendo por base o ano de competência de $2012^{23}$.

Em relação ao desenvolvimento humano (o IDH-M e IPDM), foi acessado pelas bases de dados disponíveis no PNUD e Ipardes ${ }^{24,25}$.

Para estabelecer a correlação entre os indicadores de saúde bucal, foram utilizados os dados referentes a 2012 em comparação com os índices de desenvolvimento humano (IDH-M e IPDM), de 2010. Na análise estatística quantitativa, foi utilizado o programa BioEstat 5.3, no qual foi realizado o teste de correlação de Pearson, com nível de significância de $95 \%$. 


\section{Resultados}

Em relação ao IDH-M, em 2010, constatou-se que dez municípios do Núcleo Urbano Central (NUC) da Região Metropolitana de Curitiba, encontram-se entre 0,700 e 0,799 , sendo, portanto, considerados de alto desenvolvimento humano. E três municípios (Almirante Tamandaré, Itaperuçu e Rio Branco do Sul) apresentavam IDH-M entre 0,600 e 0,699 , o que constata um médio desenvolvimento humano. Apenas um município (Curitiba) apresentou IDH-M maior que 0,800 , o qual é considerado muito alto. Já no que tange ao IPDM, no ano de 2010, três municípios (Itaperuçu, Piraquara e Rio Branco do Sul), segundo Ipardes ${ }^{17}$ (2010) eram considerados de médio-baixo desempenho municipal. A capital do estado é o único município do NUC classificado de alto desempenho, e o restante dos municípios estão compreendidos entre 0,600 a 0,799 ; o que os qualificam em médio desempenho (Tab. 1).

Tabela 1 - Índices de Desenvolvimento Humano (IDH-M e IPDM) dos municípios do NUC da Região Metropolitana de Curitiba, 2013

\begin{tabular}{|c|c|c|}
\hline Município & IDH-M (2010) & IPDM (2010) \\
\hline Almirante Tamandaré & 0.699 & 0.6029 \\
\hline Araucária & 0.740 & 0.7875 \\
\hline Campina Grande do Sul & 0.718 & 0.6788 \\
\hline Campo Largo & 0.745 & 0.7372 \\
\hline Campo Magro & 0.701 & 0.7002 \\
\hline Colombo & 0.733 & 0.6733 \\
\hline Curitiba & 0.823 & 0.8691 \\
\hline Fazenda Rio Grande & 0.720 & 0.6454 \\
\hline Itaperuçu & 0.637 & 0.5386 \\
\hline Pinhais & 0.751 & 0.7547 \\
\hline Piraquara & 0.700 & 0.5849 \\
\hline Quatro Barras & 0.742 & 0.7776 \\
\hline Rio Branco do Sul & 0.679 & 0.5979 \\
\hline São José dos Pinhais & 0.758 & 0.7558 \\
\hline
\end{tabular}

Em relação à média de primeira consulta odontológica programática, os municípios de Araucária, Campina Grande do Sul, Curitiba e Quatro Barras obtiveram os melhores indicadores em 2012. Os municípios Itaperuçu, Pinhais e Rio Branco do Sul apresentaram, respectivamente, os piores resultados (Tab. 2).
Tabela 2 - Indicadores de saúde bucal nos municípios do NUC da Região Metropolitana de Curitiba no ano de 2012

\begin{tabular}{|c|c|c|c|c|}
\hline Município & $\begin{array}{c}\text { Média 1aㅡ } \\
\text { consulta } \\
\text { programáti- } \\
\text { ca/hab. }\end{array}$ & $\begin{array}{c}\text { Escovação } \\
\text { Supervisio- } \\
\text { nada/hab. }\end{array}$ & $\begin{array}{c}\text { Média de } \\
\text { Procedimen- } \\
\text { tos indivi- } \\
\text { duais/hab. }\end{array}$ & $\begin{array}{c}\text { Média de } \\
\text { Exodontia/ } \\
\text { hab. }\end{array}$ \\
\hline $\begin{array}{l}\text { Almirante } \\
\text { Tamandaré }\end{array}$ & 0.04 & 1.52 & 0.16 & 0.02 \\
\hline Araucária & 0.17 & 0.97 & 0.70 & 0.08 \\
\hline $\begin{array}{l}\text { Campina } \\
\text { Grande do Sul }\end{array}$ & 0.11 & 0.12 & 0.37 & 0.00 \\
\hline Campo Largo & 0.06 & 0.23 & 0.21 & 0.02 \\
\hline Campo Magro & 0.06 & 0.01 & 0.34 & 0.03 \\
\hline Colombo & 0.04 & 0.12 & 1.30 & 0.02 \\
\hline Curitiba & 0.09 & 0.40 & 0.42 & 0.04 \\
\hline $\begin{array}{l}\text { Fazenda Rio } \\
\text { Grande }\end{array}$ & 0.06 & 0.43 & 0.17 & 0.00 \\
\hline Itaperuçu & 0.01 & 0.32 & 0.01 & 0.01 \\
\hline Pinhais & 0.02 & 0.33 & 0.19 & 0.01 \\
\hline Piraquara & 0.05 & 0.03 & 0.20 & 0.03 \\
\hline Quatro Barras & 0.09 & 0.00 & 0.66 & 0.06 \\
\hline $\begin{array}{l}\text { Rio Branco } \\
\text { do Sul }\end{array}$ & 0.03 & 0.49 & 0.19 & 0.03 \\
\hline $\begin{array}{l}\text { São José dos } \\
\text { Pinhais }\end{array}$ & 0.05 & 0.04 & 0.40 & 0.02 \\
\hline
\end{tabular}

Fonte: SIA-SUS (2012)23

Quanto à escovação supervisionada, observou-se que os municípios de Almirante Tamandaré e Araucária apresentam os melhores indicadores. Em contrapartida, Quatro Barras, Campo Magro e Piraquara apresentam os indicadores mais baixos (Tab. 2).

Em se tratando da média de procedimentos individuais, os municípios de Colombo, Araucária e Quatro Barras apresentam os melhores indicadores. O primeiro apresenta o indicador acima do valor 1,0. Em oposição, o município de Itaperuçu apresenta o pior indicador $(0,01)$. Em relação ao número de exodontias, encontraram-se valores relativamente baixos para todos os municípios (Tab. 2).

Na Figura 1, verificou-se uma correlação positiva entre o IPDM e a média de primeira consulta programática por habitante. Observou-se, ainda, uma correlação positiva entre a primeira consulta odontológica programática/hab. e média de exodontias/hab. (Figura 2). 


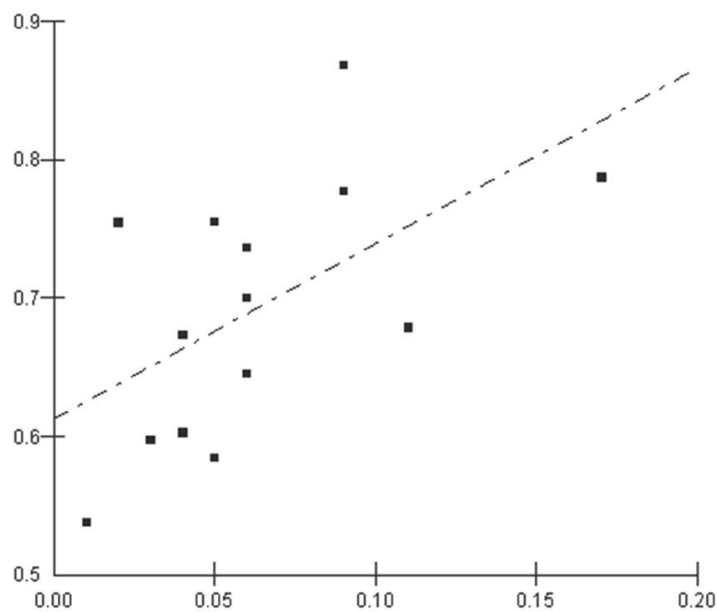

Figura 1 - Correlação entre Desenvolvimento Humano (IPDM) e Primeira Consulta Odontológica Programática/hab. no ano de 2012 nos municípios do NUC da Região Metropolitana de Curitiba $(p=0.0354 ; r=0.5645)$

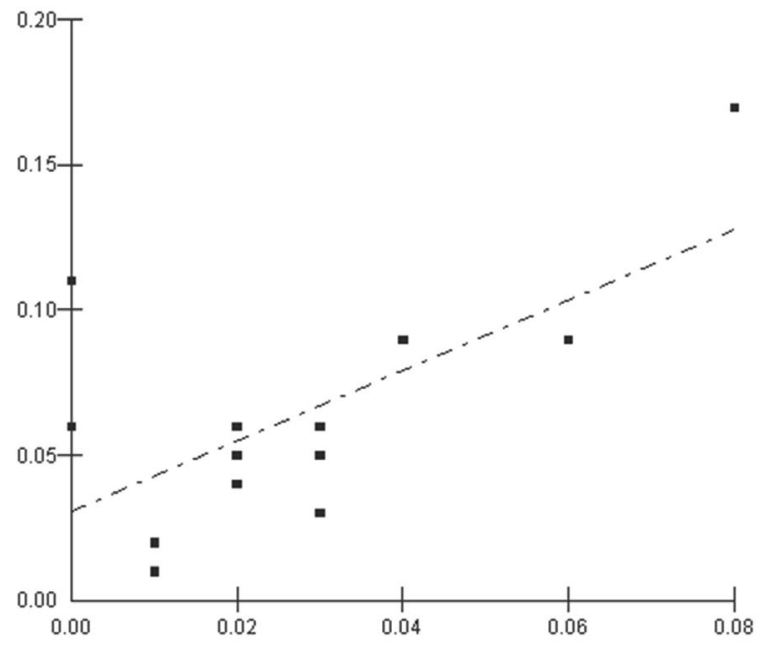

Figura 2 - Correlação entre primeira consulta odontológica programática/hab. e média de exodontias/hab. no ano de 2012 nos municípios do NUC da Região Metropolitana de Curitiba $(p=0.0129 ; r=0.6439)$

\section{Discussão}

Assim como se verifica em outros trabalhos que utilizam dados secundários, o grande problema enfrentado é a falta de confiabilidade nos dados analisados, uma vez que nem sempre os registros dos procedimentos nas bases de dados são feitos de forma precisa pelos municípios. O SIA-SUS foi implantado, inicialmente com a finalidade de pagamento dos procedimentos realizados, o que podia gerar uma interferência no registro dos dados, já que, quanto maior a produção, maior seria o repasse, o que poderia implicar um sobrerregistro dos procedimentos nos primeiros anos. No entanto, essa realidade mudou quando o repasse financeiro deixou de estar atrelado ao registro da produção ambulatorial, através do advento do Piso da Atenção Básica (PAB), o qual estabeleceu um valor per capita para o custeio das ações e serviços da Atenção Básica ${ }^{4}$.
Observa-se uma inconsistência nos registros e no controle da qualidade dessas informações nas esferas municipais, estaduais e federal, a qual gera uma necessidade de um melhor treinamento dos profissionais envolvidos no repasse de dados, bem como de maior regularidade na alimentação do sistema ${ }^{9,12,26,27}$. Ainda assim, cabe ressaltar a importância da utilização desses dados enquanto ferramenta de avaliação, de monitoramento e de planejamento das ações de serviços de saúde bucal ${ }^{8,12,28}$.

Percebeu-se, em relação à média de primeira consulta odontológica programática, que os municípios de Araucária, Campina Grande do Sul, Curitiba e Quatro Barras obtiveram os melhores indicadores em 2012. Quando comparado com a pesquisa de Stahlhoefer e Onuki $(2012)^{12}$ com os dados de 2011, observou-se que os indicadores desses quatro municípios apresentaram uma redução. Os municípios Itaperuçu, Pinhais e Rio Branco do Sul apresentaram, respectivamente, os piores resultados, o que demonstra que suas redes de serviços de saúde bucal devem priorizar a demanda espontânea e as atividades educativas e preventivas em detrimento dos procedimentos clínicos, o que comprova a baixa realização de consultas com finalidade de diagnóstico e de tratamento (Tab.2). Desse modo, o melhor resultado na média da primeira consulta odontológica programática pode estar associada à cobertura da Equipe de Saúde Bucal (ESB) na Estratégia de Saúde da Família (ESF). Marques et al. ${ }^{29}$ (2013) constataram, nos mesmos municípios do NUC na região metropolitana de Curitiba-PR, com os dados do SIA-SUS de 2012, que quanto maior é a cobertura das Equipes de Saúde Bucal na ESF, maior é o número de primeira consulta odontológica programática $(P=0.0018 ; \mathrm{r}=0.7555)$.

Quanto à escovação supervisionada, observou-se que os municípios de Almirante Tamandaré e Araucária apresentam os melhores indicadores. Em contrapartida, Quatro Barras, Campo Magro e Piraquara apresentam os indicadores mais baixos. Uma possível justificativa para esse fato é que a prática de realização de procedimentos coletivos nesses últimos municípios não é considerada relevante à saúde da população. Assim, são, em geral, incorretamente registrados, ou, até mesmo, não realizados de forma padronizada ${ }^{26}$.

O município de Itaperuçu apresenta o pior indicador em relação à média de procedimentos individuais. Uma possível justificativa para esse baixo indicador seria a prioridade dada pelo município à realização de procedimentos coletivos.

Ao avaliar a média dos quatro indicadores nos municípios estudados, pode-se observar que os valores encontram-se abaixo do ideal, o qual seria correspondente a valores mais próximos de 1,0. Isso significaria um maior atendimento assistencial à população coberta ${ }^{26}$.

A correlação positiva entre o IPDM e a média de primeira consulta programática por habitante, 
indica que municípios com melhor desenvolvimento humano apresentam maior preocupação com o agendamento de consultas com finalidade de diagnóstico e de elaboração de plano de tratamento. Fischer et al. ${ }^{30}(2010)$ ao avaliar a região Sul do Brasil, também constataram que os municípios com maiores índices de desenvolvimento humano apresentaram maior proporção do indicador de primeira consulta programática, o que pode ser explicado pelo fato de os municípios com melhor desenvolvimento humano apresentarem melhor provisão de serviços. O presente estudo, no entanto, encontra-se em discordância com Stahlhoefer e Onuki ${ }^{12}$ (2012), uma vez que, em sua pesquisa contendo os indicadores de saúde bucal do ano de 2011, encontraram correlação positiva entre o IPDM e a escovação supervisionada, não encontrando correlação entre o IPDM e a primeira consulta programática. Dessa forma, comprovando que os municípios do NUC da região metropolitana de Curitiba-PR veem avançando com melhores resultados no decorrer dos anos no indicador média de primeira consulta odontológica programática.

A correlação positiva encontrada entre a primeira consulta odontológica programática/hab. e média de exodontias/hab. comprova que municípios que realizam maior número de consultas odontológicas com finalidade de diagnóstico e de plano de tratamento são aqueles que realizam maior número de exodontias por habitante.

O resultado do presente estudo corrobora o apresentado por Stahlhoefer e Onuki ${ }^{12}$ (2012) em sua pesquisa contendo os dados de 2011, o que evidencia uma manutenção da correlação existente por dois anos consecutivos. Em contraposição, difere do resultado encontrado por Fernandes e Peres $^{31}$ (2005), em que se constatou uma correlação negativa entre o indicador de exodontias e o de cobertura de consultas odontológicas, ou seja, quanto maior o número de primeiras consultas, menor o número de exodontias.

A análise do indicador da média de exodontias se mostra bastante relevante para demonstrar o modelo de saúde adotado pelo município, esclarecendo se esse está focado na promoção de saúde ou se desenvolve somente em aspectos mutiladores. O comportamento desse indicador pode, portanto, revelar a falta de acesso da população aos serviços odontológicos, o tipo de prática desenvolvida pelos profissionais, a gestão desenvolvida no município, bem como as características culturais de determinadas comunidades ${ }^{26}$. No presente estudo, embora tenha havido correlação entre os indicadores, observou-se um baixo indicador de média de exodontias por habitante, levando em consideração que o valor mais alto encontrado foi de 0,08 .

Ao contrário do que se encontra na literatura, no presente estudo não se encontrou uma correlação negativa entre o indicador de exodontias e os indicadores socioeconômicos, mas observaram-se algumas associações negativas na análise individual dos municípios. Palmier et al. ${ }^{28}$ (2012) verificaram que a proporção de exodontias apresenta importante relação com desigualdades na distribuição de renda e da cobertura da Estratégia Saúde da Família. Já Marques et al. ${ }^{29}$ (2013) constataram, nos mesmos municípios do NUC na região metropolitana de Curitiba-PR, que quanto maior é a cobertura das Equipes de Saúde Bucal na ESF, maior também é a média de exodontias/hab. $(P=0.0101 ; \mathrm{r}=0.6603)$.

Cabe ainda ressaltar a fragilidade do Indicador de Média de Primeira Consulta Programática, o qual teve correlação com o IPDM e com a média de Exodontias. Isso ocorre, segundo Junqueira et al. ${ }^{32}(2012)$, porque embora a intenção seja medir a porcentagem da população que teve acesso ao cirurgião-dentista do serviço público, muitas vezes, esse valor é superestimado, uma vez que são incorporados ao indicador os atendimentos de emergência realizados na primeira consulta. Para a odontologia, isso não representa intervenção adequada para a recuperação e controle das doenças bucais e, para os serviços, não demonstra resolutividade, pois o paciente não retorna para completar o tratamento.

Facchini et al. ${ }^{26}$ (2011) afirmam que a relação inversa apresentada pelo número de exodontias e as primeiras consultas odontológicas programáticas deve ser encarada com mais cuidado. Os autores ressaltam, ainda, que apesar da proposta ser de reorganização da Atenção Básica, o que tem acontecido é a expansão do serviço de atendimento da livre demanda, e, consequentemente, da crescente demanda reprimida de atendimento cirúrgico restaurador. Outra possibilidade para justificar a correlação existente entre a média de exodontias e o número de primeiras consultas programáticas é o elevado número de exodontias realizado nas consultas emergenciais, já que em alguns municípios esse tipo de consulta, sem continuidade de atendimento, é registrado equivocadamente como primeira consulta odontológica programática, ocorrendo um sobrerregistro nesse indicador.

Cabe destacar o entendimento de Fischer et al. ${ }^{30}$ (2010) sobre a importância desses estudos. Segundo estes, sua realização é extremamente útil para o planejamento das ações, como por exemplo, a oferta de outras possibilidades terapêuticas consideradas conservadoras, as quais têm o intuito de melhorar o indicador de exodontias e de procedimentos odontológicos individuais na atenção básica, cuja característica mutiladora se repete, mesmo em locais com maiores coberturas da Estratégia Saúde da Família. Esses achados também podem auxiliar os gestores da região estudada para reorientação ou para manutenção das políticas de saúde bucal ora vigente, de forma socialmente orientada. 


\section{Conclusão}

A realização da presente pesquisa permitiu a percepção da importância do uso dos Sistemas de Informação em Saúde (SIS) para a avaliação das ações em saúde bucal nos municípios brasileiros. Por serem unidades de produção, de análise e de disseminação de dados, permitindo a elaboração e a avaliação das ações de saúde desenvolvidas, os itens desse sistema constituem-se em importante ferramenta para a avaliação da política de saúde bucal implantada em cada município e para o planejamento de ações futuras.

Observa-se, no entanto, que o uso e o preenchimento dos dados no sistema ainda necessitam ser aperfeiçoados, uma vez que se verificou falta de confiabilidade em algumas análises, pois nem sempre os registros dos procedimentos nas bases de dados são feitos de forma precisa pelos municípios. Faz-se necessário, portanto, um melhor treinamento dos profissionais envolvidos no repasse de dados, bem como maior regularidade na alimentação do sistema para que possam ser utilizados para análises e difusão.

A análise dos dados contidos no SIA-SUS, no ano de 2012, permitiu observar que esses municípios se encontram em diferentes estágios de reorganização da atenção à saúde bucal, com o foco em suas ações nos diferentes pontos. Além disso, a comparação desses dados com os indicadores socioeconômicos explicitou uma correlação importante entre eles, principalmente no que se refere ao índice de Primeiras Consultas Programáticas, no qual, quanto maior o desenvolvimento humano, maior é o valor desse indicador, refletindo o perfil de preocupação em estabelecer o diagnóstico e o plano de tratamento dos usuários da Atenção Básica desses municípios. Essa realidade evidencia a necessidade da reorganização da atenção em saúde para reduzir a iniquidade social.

\section{Abstract}

Objective: The aim of this study is to monitor the oral health actions of the cities which compose the Central Urban Core (CUC) of the Metropolitan Region of Curitiba $-P R$, using information systems and verifying the correlation between Primary Oral Health Care and Human Development Indicators. Materials and method: This is a descriptive correlational study with evaluation of secondary data. The human development indicators used for this study were the Municipal Human Development Index (IDHM) and the IPARDES Index of Municipal Performance (IPDM), collected in the database provided by United Nations Development Programme (UNDP) and Parana Institute of Economic and Social Development (IPARDES), referring to the year 2010. The oral health indicators were accessed and analyzed by the production of the Outpatient Information System (SIA-SUS) and are related to the year of 2012. There were used four in- dicators: a) first programmatic dental visit; b) collective actions of supervised toothbrushing; c) individual basic dental procedures; and d) extractions of deciduous and permanent teeth. In quantitative statistical analysis, we used the Pearson correlation test, with a significance level of 95\%. Results: The data comparison explained that the higher is human development, the higher is the first programmatic dental visit index $(r=0.5645)$. Correlation was also found between the first programmatic dental visit and the extractions index ( $r=0.6439)$. Conclusion: Thereby, information systems are important tools in local planning of oral health actions.

Keywords: Health status indicators. Health information systems. Health information management. Oral health. Unified health system.

\section{Referências}

1. Brasil. Ministério da Saúde. Política Nacional de Atenção Básica. Brasília: MS, 2012. 110 p.

2. Palú APN. A Inserção da Saúde Bucal no PSF, Perspectivas e Desafios: A Visão de Odontólogos do Paraná [dissertação de mestrado]. Londrina: Universidade Estadual de Londrina; 2004.

3. Volpato LER, Scatena JH. Análise da política de saúde bucal do Município de Cuiabá, Estado de Mato Grosso, Brasil, a partir do banco de dados do Sistema de Informações Ambulatoriais do Sistema Único de Saúde (SIA-SUS). Epidemiol Serv Saúde 2006; 15(2):47-55.

4. Barros SG, Chaves SCL. A Utilização do Sistema de Informações Ambulatoriais (SIA-SUS) como instrumento para caracterização das ações de saúde bucal. Epidemiol Serv Saúde 2003; 12(1):41-51.

5. Goes PSA, Moysés SJ. A utilização da informação para o planejamento e a programação em saúde bucal. In: Goes PSA de, Moysés SJ. Planejamento, gestão e avaliação em saúde bucal. São Paulo: Artes Médicas; 2012. p. 55-69.

6. Almeida GCM, Ferreira MAF. Saúde bucal no contexto do Programa Saúde da Família: práticas de prevenção orientadas ao indivíduo e ao coletivo. Cad Saúde Pública 2008; 24(9):2131-40.

7. Rio Grande do Sul. Secretaria da Saúde. Manual para sistema de informação em saúde bucal do SUS. Porto Alegre: 2007.

8. Batista SMO. Associação entre indicadores de atenção primária em saúde bucal e condições socioeconômicas e de provisão de serviços públicos odontológicos nos municípios do estado de Goiás [dissertação de mestrado]. Recife: Fundação Oswaldo Cruz, 2010.

9. Kobayashi HM. Indicadores utilizados para avaliação da assistência em saúde bucal no Brasil [monografia de especialização]. São Paulo: Universidade Federal de São Paulo, 2011.

10. Pereira CRS. Impacto da Estratégia Saúde da Família sobre indicadores de saúde bucal: análise em municípios do Nordeste brasileiro com mais de 100 mil habitantes. Cad Saúde Pública 2012; 28(3):449-62.

11. Brasília (Distrito Federal). Secretaria em Atenção à Saúde. Saúde Bucal. Brasília: 2008

12. Stahlhoefer AG, Onuki LY. O uso de sistemas de informações como estratégia de avaliação das ações de saúde bucal da região metropolitana de Curitiba-PR [Trabalho de conclusão de curso]. Curitiba: Universidade Federal do Paraná, 2013. 
13. Programa das Nações Unidas Para o Desenvolvimento (PNUD). O que é IDH. 2013 [acesso em 20 out 2013]. Disponível em: http://www.pnud.org.br/IDH/IDH. aspx?indiceAccordion=0\&li=li_IDH

14. Rio Grande do Sul. Secretaria de Planejamento, Gestão e Participação Cidadã. Índice de Desenvolvimento Humano IDH. 2013 [Acesso em: 20 out. 2013]. Disponível em: http:// www.seplag.rs.gov.br/atlas/atlas.asp?menu=439

15. Programa das Nações Unidas para o Desenvolvimento (PNUD). Atlas do desenvolvimento humano no Brasil 2013. [Acesso em: 20 out. 2013]. Disponível em: http://atlasbrasil. org.br/2013/o_atlas/idhm

16. Instituto Paranaense de Desenvolvimento Econômico e Social (IPARDES). Índice IPARDES de Desempenho Municipal: nota metodológica. Curitiba: IPARDES, 2012.

17. Instituto Paranaense de Desenvolvimento Econômico e Social (IPARDES). Índice IPARDES de Desempenho Municipal em 2010: comentários. Curitiba: IPARDES, 2010.

18. Roncalli AG. Epidemiologia das desigualdades em saúde bucal com foco em famílias. In: Moysés ST, Kriger L, Moysés SJ. Saúde bucal das famílias: Trabalhando com evidências. São Paulo: Artes Médicas; 2008. p. 170-192.

19. Correia Junior WLM. Estudo sobre procedimentos exodônticos na rede assistencial pública do Estado de Pernambuco, no período de 2000 a 2008 [Monografia de Especialização]. Recife: Fundação Oswaldo Cruz, 2012.

20. Moysés SJ. Oral health and healthy cities: an analysis of intra-urban differentials in oral health outcomes in relation to "healthy cities" policies in Curitiba, Brazil. [Thesis]. London: University College London; 2000.

21. Watt $R$, Sheiham A. Inequalities in oral health: a review of the evidence and recommendations for action. Br Dent J 1999; 187(1):6-12.

22. Coordenação de Região Metropolitana de Curitiba. Municípios da RMC 2012. [acesso em 29 out 2013]. Disponível em: http://www.comec.pr.gov.br/modules/conteudo/conteudo. php?conteudo=89

23. Brasil. Departamento de Informática do SUS. Sistema de Informações Ambulatoriais do Sistema Único de Saúde (SIA-SUS). Produção Ambulatorial do SUS - Paraná - por local de atendimento - 2008/2012. 2013a. [acesso em 30 out 2013]. Disponível em: http://www.tabnet.datasus.gov.br/cgi/ deftohtm.exe?sia/cnv/qapr.def.

24. Programa das Nações Unidas para o Desenvolvimento (PNUD). O que é Desenvolvimento Humano. 2013. [acesso em 30 out 2013]. Disponível em: http://www.pnud.org.br/IDH/DesenvolvimentoHumano.aspx?indiceAccordion=0\&li=li_DH.

25. Instituto Paranaense de Desenvolvimento Econômico e Social (IPARDES) Índice IPARDES de Desempenho Municipal - 2002/2005/2007-2010. Curitiba: IPARDES, 2013b. 7 p. [acesso em 30 out 2013]. Disponível em: http://www.ipardes. gov.br/pdf/indices/ipdm/IPDM_indice_geral_2002_2010.pdf.

26. Facchini LA, Teixeira ND, Castilho ED. Avaliação da evolução da demanda de saúde bucal através do uso de sistemas de informação em saúde. Rev Enferm Saúde 2011; 1(1):50-9.

27. Baldani MH, Almeida ES, Antunes JLF. Equidade e provisão de serviços públicos e odontológicos no Estado do Paraná. Rev Saúde Pública 2009; 43(3):446-54.

28. Palmier AC, Andrade DA, Campos ACV, Abreu MHNG, Ferreira EF. Indicadores socioeconômicos e serviços odontológicos em uma região brasileira desfavorecida. Rev Panam Salud Publica 2012; 32(1):22-9.

29. Marques AB, Oneda G, Buffon MCM, Ditterich RG. Sistemas de Informações como ferramenta de monitoramento das ações de saúde bucal na Estratégia Saúde da Família da região metropolitana de Curitiba-PR. Rev Bras Pesqui Saúde 2013.
30. Fischer TK, Peres KG, Kupek E, Peres MA. Indicadores de atenção básica em saúde bucal: associação com as condições socioeconômicas, provisão de serviços, fluoretação de águas e a estratégia de saúde da família no Sul do Brasil. Rev Bras Epidemiol 2010; 13(1):126-38.

31. Fernandes LS, Peres MA. Associação entre atenção básica em saúde bucal e indicadores socioeconômicos municipais. Rev Saúde Pública 2005; 39(6): 930-6.

32. Junqueira SR, Frias AC, Zilbovicius C, Araújo ME de. Saúde Bucal e uso dos serviços odontológicos em função do Índice de Necessidades em Saúde: São Paulo, 2008. Cien Saúde Colet 2012; 17(4):1015-24.

\section{Endereço para correspondência:}

Thábata Cristy Zermiani

Rua Antônio Vidal, 421, Moradias Bom Jesus

83602-560 Campo Largo-PR

Fone: (041) 9925-3863

E-mail: thabata.cristy@hotmail.com

Recebido: 24/01/2014. Aceito: 22/05/2014. 